\title{
Planejamento de Custos em Ambientes de Desenvolvimento de Software Orientados à Organização
}

\author{
Monalessa Perini Barcellos, Ana Regina Rocha, Guilherme Horta Travassos \\ Universidade Federal do Rio de Janeiro - COPPE/Sistemas \\ Caixa Postal 68511 - CEP 21941-972 - Rio de Janeiro, RJ - Brasil \\ (mona, darocha, ght)@cos.ufrj.br
}

\begin{abstract}
Resumo
Entregar um produto com qualidade, dentro do prazo e custos esperados é hoje um grande desafio para as organizações. Fatores como esses impulsionam o interesse, por parte das organizações, pela estimativa e controle do tempo e custos dos projetos de software. Nas últimas décadas, muitas pesquisas têm sido realizadas no sentido de desenvolver modelos para estimar prazos e custos que resultem em estimativas o mais próximo possível dos valores reais dos projetos. A utilização dos conceitos e práticas de gerência do conhecimento tem se mostrado eficiente no apoio ao planejamento de tempo e custos de projetos. Este artigo apresenta o trabalho desenvolvido em uma tese de mestrado que propõe uma abordagem para o planejamento de tempo e custos de projetos de software, baseada nas normas NBR ISO 10006, ISO/IEC DTR 16326, no guia PMBOK (Project Management Body of Knowledge), nos princípios da gerência do conhecimento e nos modelos paramétricos COCOMO II e Análise de Pontos de Função. A ferramenta CustPlan, desenvolvida para apoiar a abordagem descrita, também é apresentada.

Palavras-chave: Estimativas, Gerência do Conhecimento, Gerência de Tempo, Gerência de Custos
\end{abstract}

\begin{abstract}
Delivering a quality software product with expected cost and on time is today a great challenge for the organizations. These factors increase organizations interest development software projects costs estimation and control. In the last decades a lot of researches have been accomplished aiming the development of cost estimation models to produce estimations near by projects' real cost. Knowledge Management concepts using have been efficient to support cost planning. This paper describes an approach for projects Cost Planning used by an Enterprise-Oriented Software Development Environment. The approach is based on standards such as NBR ISO 10006, ISO/IEC DTR 16326, in the guide PMBOK (Project Management Body of Knowledge), in concepts of Knowledge Management and parametric models COCOMO II and Function Point Analysis. A tool (CustPlan) developed to support such approach is also presented.
\end{abstract}

Key Words: Estimates, Knowledge Management, Time Management, Costs Management

\section{Introdução}

As ferramentas e técnicas utilizadas no desenvolvimento de novas aplicações de software não têm sido suficientes para garantir o sucesso dos projetos. Problemas significativos ainda têm sido relatados. Observa-se, por exemplo, não aderência a baselines ${ }^{l}$, não cumprimento de orçamentos, elaboração de estimativas incorretas ou incoerentes e um número praticamente inaceitável de projetos cancelados, estagnados ou que não tenham atendido às expectativas dos clientes.

Tendo a modernidade tecnológica garantido a evolução dos recursos de apoio ao desenvolvimento de sistemas, como explicar tal fato? Talvez não seja tão difícil revelar e perceber que tal fato se dá pois muitos praticantes utilizam a tecnologia disponível, mas pouco conhecem os processos que ela apóia. Com isso, dificilmente seriam capazes de executar os processos sem a tecnologia de apoio, pois apenas utilizam o processo da tecnologia em si e não o processo genérico no qual a tecnologia se baseou para ser capaz de prover apoio automatizado [8]. Esse fato pode contribuir com os problemas anteriormente citados.

\footnotetext{
${ }^{1}$ Dados quantitativos que caracterizam o ponto de partida de um projeto[5].
} 
Para os executivos de negócio, o número de projetos sem sucesso é um dos pontos mais frustrantes do desenvolvimento de software, pois resulta em oportunidades perdidas e insatisfação de clientes. Assim sendo, além de outros fatores de qualidade, é importante que um produto de software seja desenvolvido com os recursos e cronograma previstos. O sucesso de um projeto depende muito da habilidade do gerente em estimar seus custos e prazos no início de seu desenvolvimento e controlá-los ao longo do processo de desenvolvimento.

Quando o tema em pauta são os prazos e custos do projeto, a definição e utilização de bons processos para sua gerência é de grande importância. O objetivo de processos para gerência de prazos e custos é fornecer diretrizes que devem ser seguidas para a realização das estimativas de um projeto e, com seu desenvolvimento, direcionar as atividades de acompanhamento e controle, de forma a auxiliar na proximidade entre os valores estimados e os reais.

Sendo assim, a utilização de processos para gerência de prazos e custos bem definidos pode auxiliar na realização de estimativas com menor margem de erro, indicar caminhos de acompanhamento e controle e, dessa forma, tornar menos frequentes e menores os desvios dos projetos, favorecendo o sucesso de um maior número de projetos de software.

Porém, apenas concluir um projeto no cronograma e orçamento previstos não mais indica ser este um projeto de sucesso. É necessário "agregar valor" à organização. Esse valor pode ser representado pelo conhecimento adquirido pela organização no desenvolvimento do projeto. Para trabalhar com a captura e utilização de conhecimento devem ser utilizados os conceitos e práticas da gerência do conhecimento.

A gerência do conhecimento tem sido reconhecida pelas organizações em geral como um importante fator de sucesso, uma vez que as constantes mudanças de mercado, tecnológicas e sociais exigem rápidas tomadas de decisão e atualizações em procedimentos, métodos e até estruturas organizacionais [1, 11]. A gerência do conhecimento trata a descoberta, aquisição, criação, disseminação e utilização de conhecimento, contribuindo para o constante aprendizado organizacional.

$\mathrm{O}$ crescente interesse pela gerência do conhecimento chegou recentemente à indústria de software e alguns pesquisadores têm trabalhado com a aplicação de seus conceitos em organizações que desenvolvem e mantêm software $[6,7,13,16,17]$. O conhecimento para gerência de prazos e custos de projetos de software é um exemplo de conhecimento presente nessas organizações. Durante o planejamento de um projeto de software é realizado o planejamento do tempo e dos custos do projeto, onde esforço, prazo e custos propriamente ditos são planejados. Quanto maior a experiência e conhecimento do gerente do projeto, maior será sua capacidade de realizar um bom planejamento de custos.

A Estação TABA, um projeto desenvolvido pela COPPE/UFRJ, é um meta-ambiente capaz de gerar, através de configuração e instanciação, ambientes de desenvolvimento de software (ADS) adequados às particularidades de organizações, processos de desenvolvimento e de projetos específicos. Para introduzir os conceitos e práticas da gerência do conhecimento na Estação TABA foram criados os Ambientes de Desenvolvimento de Software Orientados à Organização (ADSOrg). Esses ambientes se propõem a apoiar as atividades de Engenharia de Software, possibilitando a gerência do conhecimento que pode ser útil aos engenheiros de software ao longo dos projetos de uma organização [15]. Assim, o conhecimento de gerência de prazos e custos adquirido em projetos da organização é uma das áreas de conhecimento que deve ser gerenciada por esses ambientes. A dissertação descrita neste artigo encontra-se nesse contexto e tem como objetivo fornecer uma abordagem para a gerência de prazos e custos em ADSOrg e disponibilizar uma ferramenta de apoio à utilização da abordagem proposta. 
A seção 2 apresenta a metodologia de pesquisa utilizada para o desenvolvimento do trabalho. A seção 3 traz as definições dos principais modelos utilizados para realizar as estimativas de prazo e custos em projetos de software. A seção 4 apresenta os processos definidos para a gerência de tempo e gerência de custos de projetos de software. Na seção 5 é apresentada a ferramenta CustPlan, implementada para apoiar os processos descritos na seção 4. Na seção 6 é apresentada uma pesquisa realizada para apoiar o gerente na elaboração do cronograma e na seção 7 são realizadas as considerações finais.

\section{Metodologia de Pesquisa}

Para o desenvolvimento da dissertação, foi utilizada uma metodologia composta pelos seguintes passos:

(i) Análise da Literatura: Inicialmente, foi feita a análise da literatura disponível no que se refere à gerência de projetos, gerência de prazos, gerência de custos, técnicas de estimativas e gerência do conhecimento.

(ii) Escolha das Técnicas de Estimativas: Após o estudo da literatura, observando-se os resultados das experiências relatadas, foram escolhidos os modelos que seriam utilizados na abordagem proposta pelo trabalho. Os modelos de estimativas são apresentados na seção 3 deste artigo.

(iii) Definição dos Processos: Analisando-se os padrões para gerência de projetos, especificamente as partes relacionadas a prazos e custos, e, considerando-se as práticas e conceitos da gerência do conhecimento, foram definidos processos para gerência de custos e gerência de prazos. Os processos são descritos na seção 4 deste artigo.

(iv) Adaptação dos Processos Definidos aos Ambientes TABA : Após definidos os processos, estes foram adaptados aos ambientes TABA, considerando seus conceitos e ferramentas disponíveis.

(v) Realização de Pesquisa sobre Dependências usuais entre as Atividades do Processo de Desenvolvimento: Para apoiar o processo de gerência de tempo em ADSOrg, foi realizada uma pesquisa que resultou em um conjunto de dependências usuais entre as atividades do processo de desenvolvimento de software. Este conjunto pode ser consultado pelo gerente do projeto para a determinação das dependências e/ou paralelismo entre as atividades do processo de desenvolvimento. A pesquisa é apresentada, sucintamente, na seção 6 deste artigo.

(vi) Implementação da Ferramenta: A partir dos processos definidos foi implementada uma ferramenta (CustPlan) que apóia a execução dos processos de gerência de prazos e gerência de custos em um ADSOrg. A ferramenta é descrita na seção 5.

(vii) Validação e Análise dos Resultados: Para validar os resultados, a ferramenta está sendo aplicada a algumas organizações/projetos. As estimativas serão armazenadas e com a utilização contínua, será possível avaliar o caminho percorrido pela organização em suas estimativas, podendo também gerar análises dos pontos fortes e fracos da abordagem, promovendo sua melhoria. Poderá ser traçado um paralelo comparativo entre o que foi estimado e o que realmente foi praticado, fornecendo a margem de erro e os motivos a ela associada, permitindo-se, assim, continuar e evoluir a pesquisa. Essa etapa está sendo realizada após o término da dissertação, pois sua realização excederia em muito o tempo esperado para a dissertação de mestrado. No contexto da dissertação, foi apresentado, portanto, um exemplo de uso da ferramenta. A validação completa está sendo realizada no contexto do Projeto TABA, um projeto de longo prazo da COPPE, englobando outras ferramentas de gerência de projetos com enfoque em gestão do conhecimento. Desde o 
segundo semestre de 2003 Ambientes TABA estão em uso em pequenas e médias empresas do Rio de Janeiro, associadas à Riosoft.

\section{Modelos para Realização de Estimativas de Prazos e Custos de Projetos}

Nas últimas duas décadas, muitos estudos têm sido realizados na área de estimativas de prazos e custos para projetos de software, disponibilizando uma diversidade de técnicas para a realização dessas estimativas. Essas técnicas, de acordo com suas características, podem ser agrupadas em três tipos de modelos: (i) modelos paramétricos; (ii) estimativas baseadas em analogias; e, (iii) julgamento de especialistas.

\section{(i) Modelos Paramétricos}

Os modelos paramétricos utilizam características do projeto em modelos matemáticos e/ou algoritmos para calcular as estimativas do projeto. Alguns desses modelos, já considerados clássicos como o COCOMO II (evolução do COCOMO 81) [2] e a Análise de Pontos de Função [4] têm sua aplicabilidade constatada em experiências e práticas de muitas organizações, registradas em artigos e outras publicações.

Nos anos 80, modelos paramétricos foram utilizados e comparados em conjuntos de dados de projetos de diversos tamanhos e ambientes. Algumas das principais conclusões foram que esses modelos geravam resultados fracos quando aplicados sem calibração em outros ambientes. Essa conclusão levou à reavaliação das técnicas testadas, surgimento de novas técnicas e desenvolvimento de procedimentos de calibração para os modelos paramétricos, permitindo que cada organização possa utilizar valores diferentes para as constantes e outros parâmetros dos modelos, adquirindo-os através da análise de dados e projetos da própria organização, o que diminuiu consideravelmente a margem de erro das estimativas realizadas pelos modelos paramétricos. O COCOMO II [2] é um bom exemplo de modelo que permite calibração para cada organização em particular.

Nos anos 90, os estudos também envolveram modelos não paramétricos, baseados em algoritmos inteligentes e analogias, que são descritos a seguir.

\section{(ii) Estimativas Baseadas em Analogias}

As estimativas baseadas em analogias são métodos não paramétricos que utilizam dados históricos de outros projetos para realizar as estimativas para o projeto corrente. As analogias são realizadas levando-se em consideração características comuns aos projetos. São frequentemente utilizadas nas estimativas de custos totais do projeto quando existe uma quantidade limitada de informações detalhadas sobre ele, como por exemplo nas fases iniciais. Também são utilizadas para apoiar a distribuição do tempo e custos totais do projeto em suas fases, módulos e/ou atividades [12].

Para realizar as estimativas utilizando analogias é preciso que exista uma base de dados históricos que possa ser acessada para fornecer os dados dos projetos anteriores que serão utilizados como pontos de apoio para as estimativas dos novos projetos.

Muitas pesquisas mostraram que a realização de estimativas baseadas em analogias produz valores satisfatórios, desde que a base de dados históricos possua projetos similares ao projeto que está sendo estimado, que os critérios de determinação da similaridade entre projetos sejam bem definidos e que o algoritmo de busca por projetos similares seja eficiente.

Os bons resultados obtidos nos estudos de analogia de estimativas não vêm desacreditar os modelos paramétricos. Alguns dos estudos desenvolvidos associaram métodos paramétricos e analogias, obtendo resultados que levaram à conclusão de que uma associação 
desses dois tipos de modelos seria capaz de fornecer estimativas com uma margem de erro aceitável, ou seja, em torno de $20 \%$.

\section{(iii) Julgamento de Especialistas}

Existem organizações que não possuem uma base de dados históricos, o que as impede de realizar as estimativas dos projetos baseadas em analogias. Da mesma forma, existem aquelas onde não há experiência ou conhecimento para utilizar, de forma eficaz, algum modelo paramétrico de estimativas. Para realizar estimativas em organizações que se apresentem nessa situação, existe uma outro tipo de modelo chamado de opinião de especialistas, julgamento de especialistas ou utilização de experiência pessoal, e consiste no ato dos gerentes de projetos estimarem os valores para os projetos baseando-se em suas próprias experiências passadas. Alguns autores afirmam que essa ainda é a forma mais comumente utilizada pelas organizações e destacam que ela não é capaz de produzir dados históricos formais e, tipicamente, não apresenta regras para sua abordagem, além de não permitir calibrações para melhorar as estimativas mal realizadas, uma vez que não há padrão para sua realização.

Apesar das desvantagens dessa abordagem, muitas vezes, a utilização da experiência pessoal pode ser o caminho disponível para uma organização realizar as estimativas de seu primeiro projeto e iniciar, assim, a alimentação de uma base de dados históricos que possa ser utilizada nos projetos subsequentes. Além disso, alguns estudos mostraram que os valores de estimativas gerados por essa abordagem crescem em eficiência de maneira diretamente proporcional à experiência do gerente em estimar projetos de software.

Há, ainda, uma outra situação onde a opinião de um especialista é necessária e tem caráter de julgamento e decisão. Nela, o gerente do projeto utiliza sua experiência para decidir os valores das estimativas quando técnicas diferentes apresentam valores diferentes para as mesmas variáveis do projeto (prazo, custos e esforço) ou para ajustar os valores das estimativas propostos pelas técnicas.

Analisando os resultados das pesquisas das duas últimas décadas, estudos recentes mostram que utilizar uma combinação dos três tipos de modelos de estimativas é um bom caminho para obter estimativas acuradas, uma vez que os pontos fracos de um modelo podem ser minimizados pelos outros modelos.

\section{Processos de Gerência de Tempo e Gerência de Custos}

Os processos de gerência de tempo e gerência de custos possuem um conjunto de atividades maior que o necessário ao planejamento de tempo e custos de um projeto de software, uma vez que abrangem também as atividades relacionadas ao acompanhamento e controle do tempo e custos ao longo do processo de desenvolvimento.

Os processos apresentados a seguir foram definidos tendo como base a literatura de gerência de tempo e custos, as recomendações da norma NBR ISO 10006 (2000) [9], que define diretrizes para a qualidade no gerenciamento de projetos, o relatório técnico 16326 da ISO/IEC (1999) [3], que provê um guia para a aplicação da norma ISO/IEC 12207 (1995) à gerência de projetos de software, e o PMBOK (Project Management Body of Knowledge 2000) [12], o padrão para gerência de projetos publicado pelo PMI (Project Management Institute).

A norma NBR ISO 10006 (2000) [9] recomenda que sejam utilizados, em todas as atividades do processo, a experiência e dados históricos provenientes de projetos anteriores. Dessa forma, os processos aqui descritos buscam a reutilização do conhecimento e experiência organizacionais, um dos benefícios visados pela gerência do conhecimento. 
Outra observação importante é que há uma interação dos processos de gerência de tempo, gerência de custos e alocação de recursos. $\mathrm{Na}$ abordagem aqui apresentada, a gerência de tempo propõe o cronograma do projeto. Posteriormente, os recursos são alocados às atividades do cronograma e, em seguida, os custos do projeto são calculados.

\section{Processo de Gerência de Tempo}

A gerência de tempo tem como objetivo elaborar e controlar o cronograma do projeto.

Esse processo deve ser utilizado em dois momentos distintos do projeto: inicialmente, quando pouco é conhecido do projeto, onde são geradas as estimativas iniciais ainda com uma abrangência macroscópica, para realizar o planejamento do projeto e, posteriormente, quando mais informações do projeto forem obtidas, o que permite o refinamento das estimativas geradas.

No momento de realizar as primeiras estimativas, apenas as atividades do processo de desenvolvimento são analisadas. No refinamento das estimativas, as sub-atividades do processo também são consideradas.

O processo de gerência de tempo é composto por cinco atividades: (i) Identificar as dependências entre as atividades do projeto; (ii) Estimar a duração das atividades do projeto com abordagem top-down; (iii) Estimar a duração das atividades do projeto com abordagem bottom-up; (iv) Elaborar o cronograma do projeto; e , (v) Controlar o cronograma do projeto.

(i) Identificar as dependências entre as atividades do projeto: Nessa atividade as inter-relações, interações lógicas e interdependências entre as atividades do projeto são identificadas e analisadas quanto à sua consistência, pois existem atividades que podem ser realizadas independentemente de outras, mas há aquelas que precisam de uma relação de dependência temporal com outra(s).

(ii) e (iii) Estimar a duração das atividades do projeto: Para realizar as estimativas do projeto, uma abordagem deve ser escolhida pelo gerente do projeto: top-down ou bottom-up. As atividades (ii) e (iii) do processo de gerência de tempo são, então, realizadas de forma alternativa. $\mathrm{Na}$ abordagem top-down, as estimativas de prazo e esforço do projeto são realizadas, inicialmente, utilizando-se modelos paramétricos. Os valores das estimativas fornecidos pelos modelos paramétricos são, então, ajustados pelo gerente do projeto analisando-se dados de projetos similares e utilizando sua experiência pessoal para decidir as estimativas do projeto. Definidos os valores das estimativas, estes devem ser distribuídos pelas atividades do projeto. Na abordagem bottom-up são utilizados dados de projetos similares para realizar as estimativas das atividades do projeto. As estimativas totais do projeto são obtidas através do somatório das estimativas de suas atividades.

(iv) Elaborar o cronograma do projeto: Realizadas as estimativas para as atividades do projeto, o cronograma deve ser elaborado. Neste momento, são identificados os caminhos críticos do projeto, determinadas as datas de início e fim das atividades e registrados os marcos e pontos de controle no cronograma do projeto.

(v) Controlar o cronograma do projeto: Durante o desenvolvimento do projeto, o cronograma deve ser comparado, analisado e revisto sempre que necessário. Para isso, o gerente registra os desvios ocorridos e revê o cronograma, considerando a possibilidade de recuperação do desvio ou negociando com as partes envolvidas. As decisões e ações corretivas a serem tomadas só devem ser feitas após consideradas suas implicações para o projeto. As alterações do cronograma devem ser comunicadas às partes envolvidas no projeto.

\section{Processo de Gerência de Custos}

A gerência de custos tem como objetivo elaborar e controlar o orçamento do projeto. 
Este processo deve ser utilizado em momentos distintos: inicialmente, após serem realizadas as estimativas iniciais de tempo e esforço, são geradas as estimativas iniciais de custos ainda com uma abrangência macroscópica. Posteriormente, as estimativas realizadas são detalhadas e, quando as estimativas de tempo, esforço ou alocação de recursos forem revistas, as estimativas de custos também deverão ser.

O processo de gerência de custos é composto por três atividades: (i) Estimar custos do projeto; (ii) Elaborar o orçamento do projeto; e, (iii) Controlar o orçamento do projeto.

(i) Estimar custos do projeto: O primeiro passo do processo de gerência de custos é estimar os custos do projeto. Nesta atividade, os elementos de custos do projeto (recursos de hardware, recursos de software, recursos humanos e outras despesas) são identificados e suas respectivas quantidades e custos atribuídos.

(ii) Elaborar orçamento do projeto: Os valores realizados na atividade anterior são utilizados para elaborar o orçamento do projeto, que será o baseline dos custos ${ }^{2}$ para medir o desempenho do projeto. Todos os elementos de custos e seus respectivos valores, identificados/registrados anteriormente, são inseridos no orçamento do projeto com suas respectivas datas e frequência. As receitas previstas para o projeto também são registradas.

(iii) Controlar orçamento do projeto: Durante o desenvolvimento do projeto, o gerente deve comparar, analisar e rever o orçamento sempre que necessário. O controle de custos envolve identificar e documentar o motivo das variações, tanto positivas quanto negativas e adequar o orçamento a elas. Assim, os desvios ocorridos são registrados e o orçamento é alterado para adequar-se a eles. Caso os desvios registrados e/ou as ações corretivas tomadas indiquem situações de risco ao projeto, estas devem ser comunicadas às partes interessadas.

\section{A Ferramenta CustPlan}

Buscando-se apoiar a abordagem de planejamento de tempo e custos em ADSOrg na Estação TABA foi implementada a ferramenta CustPlan, que apóia as atividades presentes nos processos de gerência de tempo e gerência de custos apresentados na seção 4.

A CustPlan é disponibilizada em um ADSOrg instanciado e, dessa forma, possibilita a utilização do conhecimento organizacional armazenado no repositório da organização. Ela faz parte das ferramentas disponibilizadas ao usuário do ADSOrg durante as atividades de gerência do projeto. A CustPlan é acessada pelo gerente do projeto em momentos distintos durante o processo de desenvolvimento do projeto. Para realizar o planejamento inicial do projeto, o gerente acessará a CustPlan através da atividade Planejamento do Projeto, mais especificamente durante a realização das sub-atividades Planejamento de Tempo e Planejamento de Custos. Para refinar/detalhar as estimativas realizadas, o gerente do projeto acessará a CustPlan novamente após a especificação de requisitos do sistema estar concluída.

Como a CustPlan trata dois processos distintos, ela será dividida em duas partes: gerência de tempo e gerência de custos, para ser melhor apresentada.

\section{Gerência de Tempo}

A figura 1 apresenta a interface básica da ferramenta quando esta provê apoio ao planejamento de tempo. No lado esquerdo pode-se identificar o processo de gerência de tempo e no lado direito da interface identifica-se a atividade que está sendo realizada pelo gerente. Os ícones localizados no canto superior direito da tela permitem a realização de consulta e registro de conhecimento pertinentes às atividades do processo. $\mathrm{O}$ gerente do

\footnotetext{
${ }^{2}$ Baseline dos Custos é o orçamento referencial que será utilizado para medir e monitorar o desempenho dos custos do projeto. É desenvolvido através da totalização das estimativas de custos por período.
} 
projeto pode consultar o conhecimento registrado por outros gerentes de projetos e registrar o conhecimento adquirido por ele durante a execução da atividade.

Ao longo dos processos de gerência de tempo e custos, a CustPlan disponibiliza o conhecimento explícito armazenado para a atividade que está sendo realizada pelo gerente. A figura 2 apresenta a tela de consulta ao conhecimento armazenado no repositório da organização, ilustrando a consulta ao conhecimento armazenado para a atividade Identificar dependências entre as atividades do projeto, do processo de gerência de tempo.

$\mathrm{Na}$ parte inferior da figura 1 é possível observar a consulta às pré-atividades da atividade que está sendo analisada pelo gerente do projeto. Essa consulta acessa o conhecimento especialista armazenado no repositório da organização. Esse conhecimento foi obtido através dos resultados da pesquisa mencionada na seção 2 , que forneceu o conjunto de dependências usuais entre as atividades do processo de desenvolvimento de software, segundo a visão de gerentes de projetos entrevistados. Esta pesquisa será melhor descrita na seção 6 deste artigo.

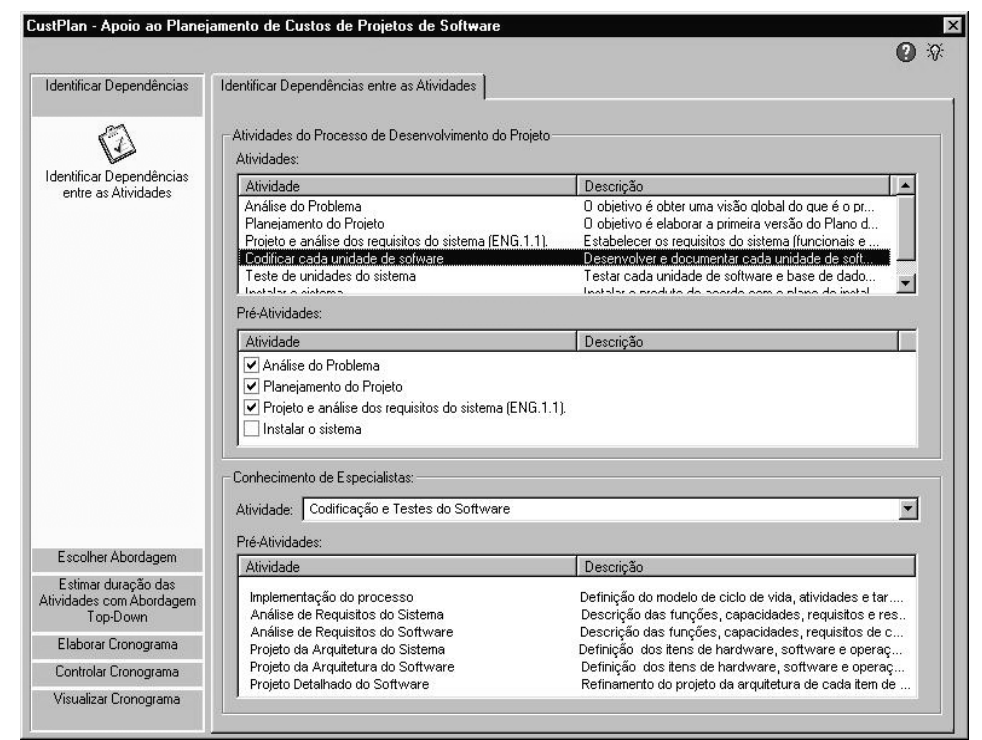

Figura 1 - Interface CustPlan - Processo de Gerência de Tempo

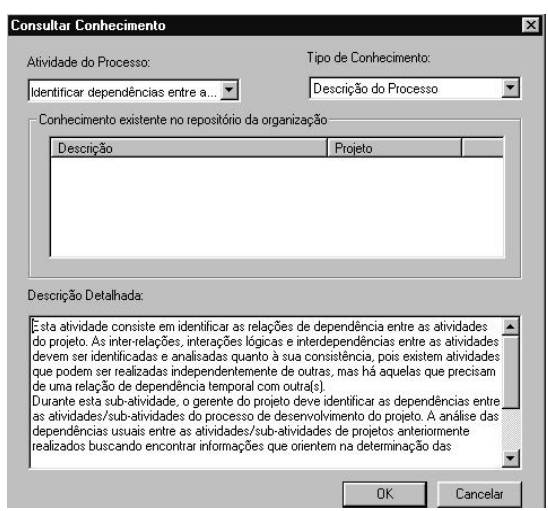

Figura 2 - Consulta ao conhecimento armazenado no repositório da organização

Conforme mencionado anteriormente, a associação de modelos diferentes para realizar as estimativas de projetos tem-se mostrado o caminho mais eficiente. Baseando-se nesses resultados, a CustPlan permite que o gerente realize as estimativas utilizando modelos paramétricos, analogia de estimativas e que utilize o conhecimento organizacional e sua 
experiência pessoal para decidir os valores das estimativas do projeto. Para associar todos esses modelos, o gerente deve usar a abordagem top-down para a realização das estimativas.

A utilização dos modelos paramétricos é realizada na execução das atividades Realizar estimativas do projeto utilizando Análise de Pontos de Função e Realizar estimativas do projeto utilizando COCOMO II, onde o gerente fornece os dados necessários ao cálculo das estimativas.

A analogia de estimativas e julgamento de especialistas são realizadas na atividade Ajustar estimativas do projeto a partir de projetos similares. A figura 3 apresenta a tela onde o gerente visualiza as estimativas calculadas pelos modelos paramétricos, realiza a busca por projetos similares e decide as estimativas do projeto.

A distribuição dos valores das estimativas do projeto pelas atividades é feita através da atividade Distribuir o tempo entre as atividades do projeto, onde o gerente poderá analisar as atividades de projetos similares anteriores para distribuir as estimativas do projeto.

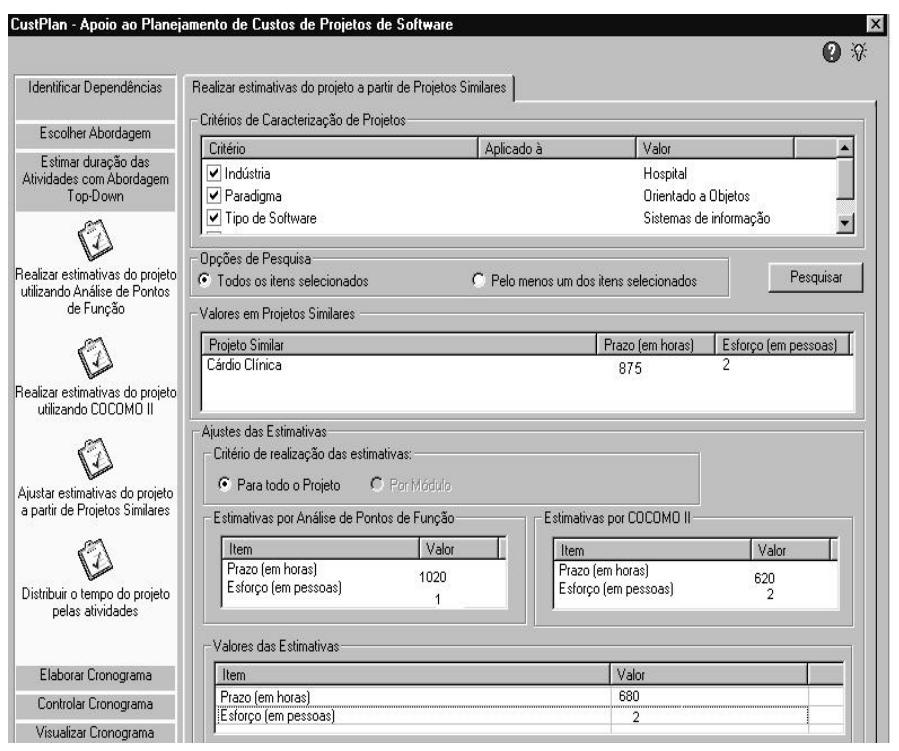

Figura 3 - Ajuste das estimativas obtidas pelos modelos paramétricos utilizando analogias e julgamento de especialista - Abordagem top-down

Caso o gerente opte pela abordagem bottom-up para realizar as estimativas do projeto, ele utilizará dados de projetos anteriores para realizar as estimativas das atividades. As estimativas totais do projeto serão calculadas pelo somatório das estimativas das atividades.

A figura 4 mostra a realização da estimativas com abordagem bottom-up. Na parte superior da tela é realizada a busca por projetos similares e, para cada atividade do projeto que está sendo estimado, são apresentadas suas estimativas nos projetos anteriores.

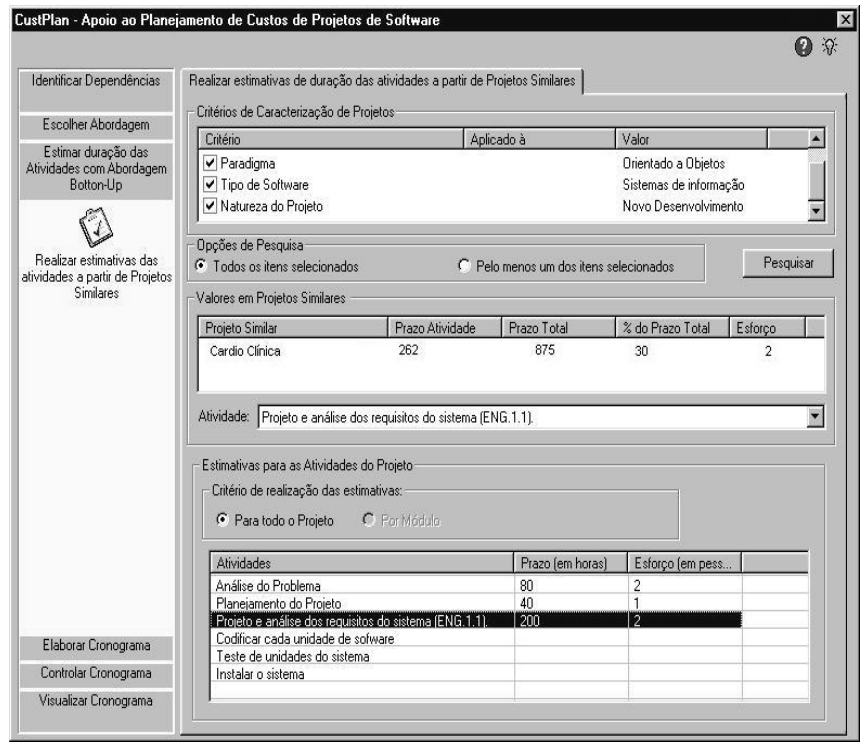

Figura 4 - Realização das estimativas para as atividades analisando dados de projetos similares - Abordagem bottom-up 
Para caracterizar os perfis dos projetos e, assim, agrupá-los como similares, foi definido um conjunto de critérios que são analisados no momento da busca por projetos similares, sendo eles: indústria; tipo de software; paradigma; natureza do projeto; nível de experiência dos gerentes, da equipe e dos clientes; distribuição geográfica da equipe; restrições de cronograma, desempenho, tempo de execução, segurança e recursos humanos; e, uso de tecnologia inovadora.

A elaboração do cronograma é feita com o apoio da CustPlan que identifica automaticamente os caminhos críticos do projeto analisando as durações e dependências entre as atividades do projeto e registra no cronograma os marcos e pontos de controle identificados no Plano de Acompanhamento do projeto. O gerente deve, então, indicar as datas de início e fim das atividades do projeto.

Para controlar o cronograma, o gerente do projeto utiliza a CustPlan para registrar os desvios que ocorreram e, posteriormente, analisa esses desvios para alterar o cronograma. A figura 5 apresenta a tela de registro dos desvios e a figura 6 apresenta a tela de alteração do cronograma.

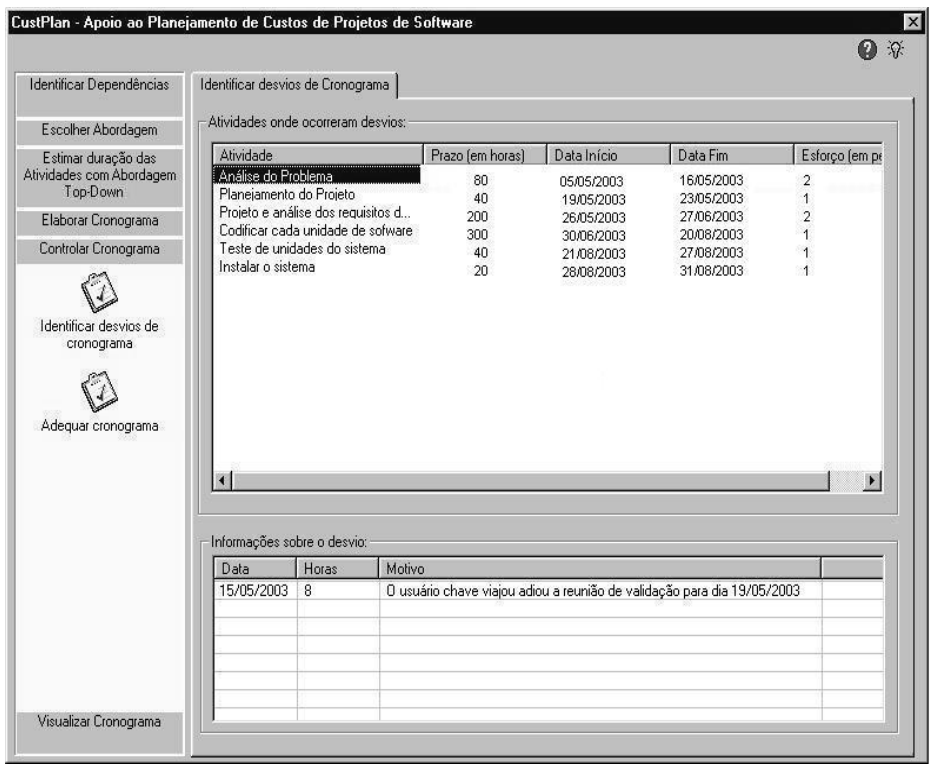

Figura 5 - Registro de desvios de cronograma

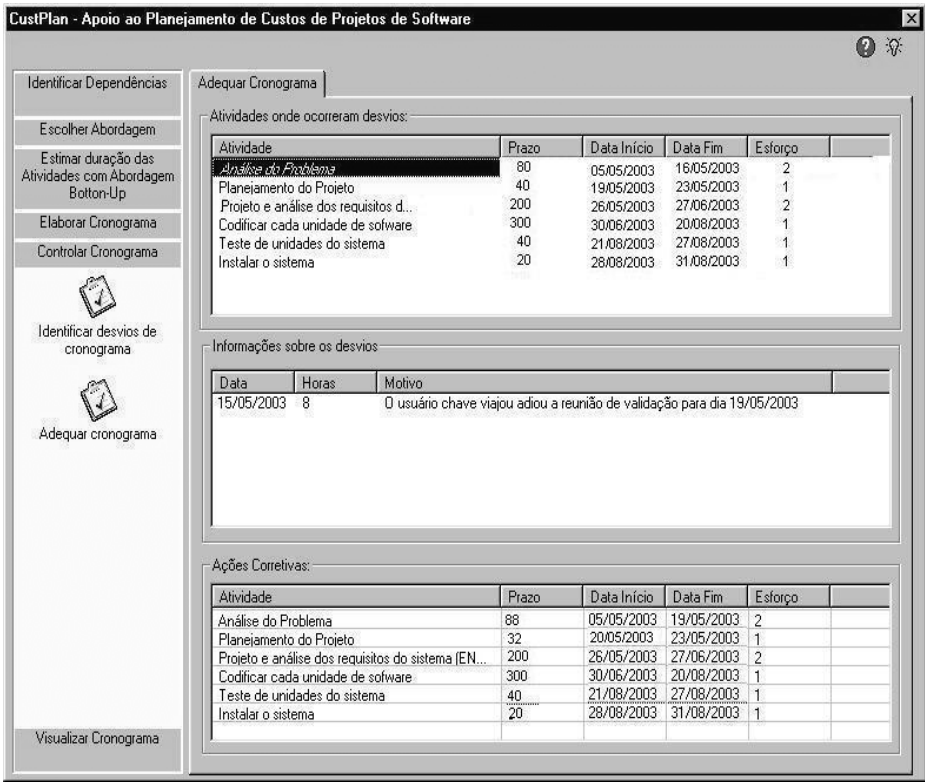

Figura 6 - Alteração de cronograma analisando desvios 


\section{Gerência de Custos}

Para apoiar o planejamento de custos, a CustPlan mantém o mesmo padrão de interface do planejamento de tempo, porém disponibilizando, neste momento, as atividades do processo de gerência de custos.

Após ser realizado o planejamento do tempo do projeto, utilizando a CustPlan, é feita a alocação de recursos humanos ao projeto, que é realizada com o apoio da ferramenta RHPlan [14], disponível na Estação TABA. Após os recursos humanos estarem alocados ao projeto, deve ser elaborado o orçamento do mesmo. Para isso, a CustPlan deve ser novamente utilizada, porém, agora, acessando o processo de gerência de custos.

Inicialmente, os elementos de custos devem ser identificados através da atividade Identificar Elementos de Custos. O gerente indica em checklists quais serão os recursos utilizados no projeto e sua quantidade. Para os recursos humanos, isso ocorre automaticamente na ferramenta, considerando as informações da alocação de recursos humanos ao projeto e o cronograma.

Em seguida, o orçamento é elaborado. Os custos com recursos humanos são calculados automaticamente por atividade do projeto, considerando os dados do cronograma e da alocação de recursos às atividades do projeto. Para os demais recursos, o gerente deve indicar a data em que a despesa ocorrerá e sua frequência. O gerente indica também as receitas previstas para o projeto.

$\mathrm{O}$ controle do orçamento é realizado de forma similar ao controle do cronograma. O gerente registra os desvios e altera o orçamento analisando os desvios registrados. A CustPlan realiza as alterações do cronograma no orçamento, automaticamente. A figura 7 mostra um exemplo de desvio de cronograma que é registrado também no orçamento. O registro do desvio no cronograma foi ilustrado na figura 5. A figura 8 mostra o orçamento alterado para adequar-se às alterações do cronograma, provocadas pelas ações corretivas ao desvio registrado.

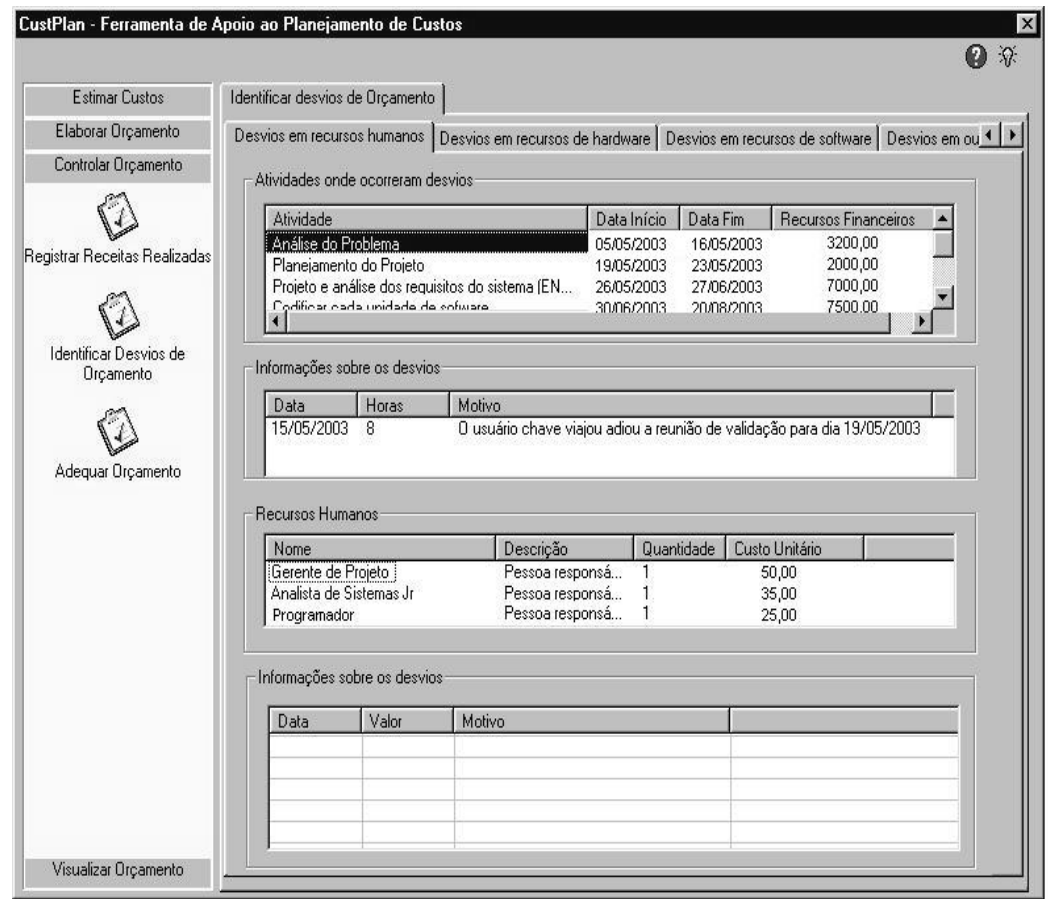

Figura 7 - Registro de desvios de cronograma no orçamento 


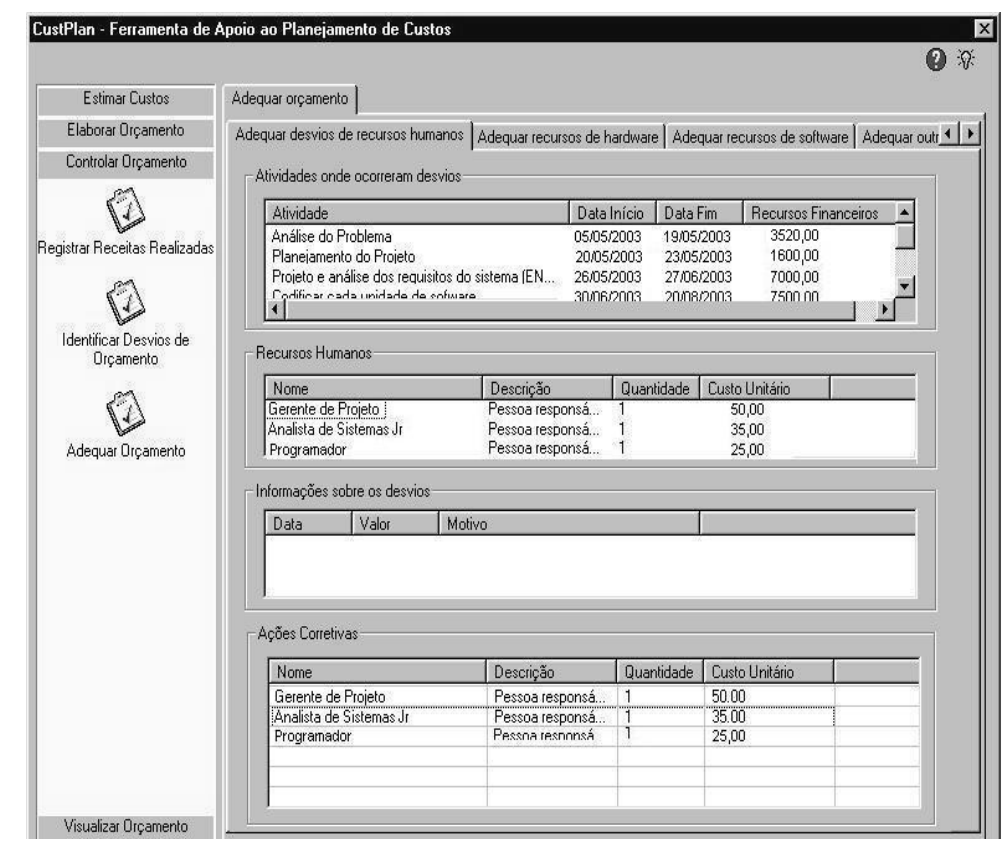

Figura 8 - Alteração de orçamento devido a desvios de cronograma

\section{Pesquisa de Dependências Usuais entre as Atividades do Processo de Desenvolvimento}

O primeiro passo para realizar o planejamento do cronograma do projeto é indicar as dependências entre as atividades do projeto, conforme apresentado no processo de gerência de tempo, descrito na seção 4. Para realizar esse passo utilizando CustPlan, conforme mostrado na figura 1 (seção 5), o gerente de projetos poderá consultar no repositório da organização as dependências usuais entre as atividades do processo de desenvolvimento.

Para coletar as dependências usuais, foi realizada uma pesquisa cujos participantes foram gerentes de projetos de software da Fundação COPPETEC (Coordenação de Projetos, Pesquisas e Estudos Tecnológicos) e outros gerentes de projetos que atuam em processos de desenvolvimento do software. Foram considerados gerentes de projeto, sendo estes mestres ou doutores na área de informática e com experiência relevante em gerência de projetos de software.

A pesquisa foi realizada utilizando-se um questionário que considerou as atividades do processo de desenvolvimento da ISO/IEC 12207 - Tecnologia de Informação - Processos de ciclo de vida de software [10]. O questionário apresentou uma tabela onde o participante indicou, para cada atividade do processo, suas pré-atividades, ou seja, as atividades que precisam estar concluídas para que ela possa ser realizada. Foi permitido ao participante registrar considerações sobre as dependências e comentários em geral.

Com base nos dados coletados, um conjunto de dependências usuais entre as atividades do processo de desenvolvimento da ISO/IEC 12207 foi proposto e armazenado no repositório da organização para ser utilizado durante a atividade Identificar as Dependências entre as Atividades do Projeto realizada no planejamento do cronograma do projeto.

Para a realização dessa pesquisa foram seguidas cinco atividades: (i) definição; (ii) planejamento; (iii) operação; (iv) análise e interpretação; e (v) apresentação. O ponto de partida foi a concepção da idéia da pesquisa, onde foi avaliado se uma pesquisa era realmente necessária e apropriada para atender as questões a serem investigadas. Dando início ao processo de realização da pesquisa propriamente dito, na atividade definição a pesquisa foi definida em termos do problema pesquisado, seus objetivos e metas. Na atividade de planejamento, o projeto da pesquisa foi realizado, a instrumentação foi elaborada e as 
ameaças à perfeita execução da pesquisa foram avaliadas. Durante esta atividade foi gerado o planejamento da pesquisa. $\mathrm{Na}$ atividade operação, os questionários foram entregues aos participantes e os dados obtidos foram organizados em uma planilha visando facilitar a análise e avaliação dos dados realizada na atividade posterior de análise e interpretação. Finalmente, os resultados foram apresentados na atividade apresentação.

\section{Resultados da Pesquisa}

Os resultados da pesquisa caracterizaram um conjunto inicial de dependências usuais entre as atividades do processo de desenvolvimento da NBR ISO/IEC 12207 que foi armazenado no repositório da organização para apoiar os gerentes de projeto na identificação das dependências entre as atividades do projeto.

Para realizar a pesquisa, um conjunto inicial de dependências foi proposto, porém, os participantes da pesquisa não tiveram acesso às dependências presentes nesse conjunto.

Para obter os resultados, inicialmente, as características dos participantes da pesquisa foram analisadas e, em seguida, foram estabelecidos pesos para cada um deles, considerando o tempo de atuação em gerência de projetos, o número de projetos gerenciados, a experiência em processos de software e o conhecimento sobre a norma NBR ISO/IEC 12207 de cada participante. A análise das características e cálculo dos pesos dos participantes os classificou em dois grupos: experientes e pouco experientes.

Foi, então, estabelecido o valor do ponto de inclusão, para indicar o valor a partir do qual uma dependência identificada pelos participantes faria parte do conjunto final de dependências.

Analisadas as dependências identificadas por cada participante, foi possível perceber que os participantes pertencentes ao grupo experiente identificaram as dependências presentes no conjunto inicial proposto, mesmo sem terem tido acesso a ele. Por outro lado, alguns participantes do grupo pouco experiente identificaram muitas dependências incoerentes.

Utilizando os pesos dos participantes e o critério de inclusão de uma dependência no conjunto de dependências (valor do ponto de inclusão), o conjunto de dependências obtido foi igual ao conjunto inicialmente proposto.

A tabela 1 apresenta o conjunto de dependências usuais obtido como resultado da pesquisa. Esse conjunto de dependências usuais foi armazenado no repositório da organização e pode ser acessado pelo gerente como apoio à determinação das dependências entre as atividades do projeto.

\begin{tabular}{|c|c|c|c|c|c|c|c|c|c|c|c|c|c|}
\hline \multirow[b]{2}{*}{$\begin{array}{l}\text { Atividades do Processo de } \\
\text { Desenvolvimento da } \\
\text { NBR ISO/IEC } 12207- \\
\text { Tecnologia de Informação- } \\
\text { Processos de ciclo de vida de } \\
\text { software que devem ser } \\
\text { executadas }\end{array}$} & \multicolumn{13}{|c|}{ Atividades que precisam estar concluídas } \\
\hline & 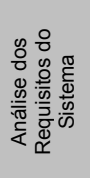 & 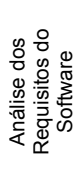 & 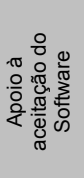 & 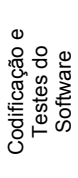 & 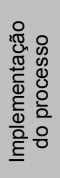 & 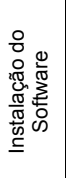 & 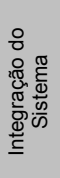 & 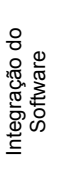 & 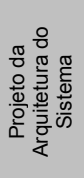 & 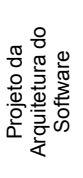 & 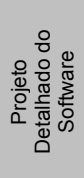 & 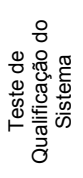 & 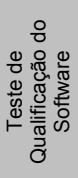 \\
\hline Análise dos Requisitos do Sistema & & & & & $\mathrm{X}$ & & & & & & & & \\
\hline Análise dos Requisitos do Software & $\mathrm{X}$ & & & & $\mathrm{X}$ & & & & & & & & \\
\hline Apoio à aceitação do Software & $\mathrm{X}$ & $\bar{X}$ & & $\mathrm{X}$ & $\mathrm{X}$ & $\mathrm{X}$ & $\mathrm{X}$ & $\mathrm{X}$ & $\mathrm{X}$ & $\mathrm{X}$ & $\mathrm{X}$ & $\mathrm{X}$ & $\mathrm{X}$ \\
\hline Codificação e Testes do Software & $\mathrm{X}$ & $\mathrm{X}$ & & & $\mathrm{X}$ & & & & $\mathrm{X}$ & $\mathrm{X}$ & $\mathrm{X}$ & & \\
\hline \multicolumn{14}{|l|}{ Implementação do processo } \\
\hline Instalação do Software & $\mathrm{X}$ & $\mathrm{X}$ & & $\mathrm{X}$ & $X$ & & $\mathrm{X}$ & $\mathrm{X}$ & $\mathrm{X}$ & $\mathrm{X}$ & $\mathrm{X}$ & $\mathrm{X}$ & $X$ \\
\hline Integração do Sistema & $\mathrm{X}$ & $\mathrm{X}$ & & $\mathrm{X}$ & $\mathrm{X}$ & & & $\mathrm{X}$ & $\mathrm{X}$ & $\mathrm{X}$ & $\mathrm{X}$ & & $\mathrm{X}$ \\
\hline Integração do Software & $\bar{X}$ & $\bar{X}$ & & $\bar{X}$ & $\mathrm{X}$ & & & & $\bar{X}$ & $\bar{X}$ & $\bar{X}$ & & \\
\hline Projeto da Arquitetura do Sistema & $\mathrm{X}$ & & & & $\mathrm{X}$ & & & & & & & & \\
\hline Projeto da Arquitetura do Software & $\bar{X}$ & $\bar{X}$ & & & $x$ & & & & $\mathrm{X}$ & & & & \\
\hline Projeto Detalhado do Software & $\mathrm{X}$ & $\mathrm{X}$ & & & $\mathrm{X}$ & & & & $\mathrm{X}$ & $\mathrm{X}$ & & & \\
\hline Teste de Qualificação do Sistema & $\mathrm{X}$ & $\mathrm{X}$ & & $\mathrm{X}$ & $\mathrm{X}$ & & $\mathrm{X}$ & $\bar{x}$ & $\mathrm{X}$ & $\mathrm{X}$ & $\mathrm{X}$ & & $x$ \\
\hline Teste de Qualificação do Software & $\mathrm{X}$ & $\mathrm{X}$ & & $\mathrm{X}$ & $\mathrm{X}$ & & & $\mathrm{X}$ & $\mathrm{X}$ & $\mathrm{X}$ & $\mathrm{X}$ & & \\
\hline
\end{tabular}

Tabela 1 - Conjunto de dependências usuais entre as atividades do processo de desenvolvimento 


\section{Considerações Finais}

A tecnologia hoje disponível para o desenvolvimento de software permite, e até induz, a utilização de arquiteturas de sistemas cada vez maiores e mais complexas. Em contrapartida, o prazo para o desenvolvimento de tais produtos tem sido compactado, refletindo a evolução tecnológica e necessidades econômicas do mercado. As dimensões dos produtos de software estão atingindo níveis quantitativos cada vez maiores, não ocorrendo o mesmo com a tolerância a falhas de previsão de orçamentos e cronogramas. Esse cenário exige que processos formalizados de gerência de projetos de software sejam definidos e utilizados.

A gerência dos prazos e custos de projetos de software é muito importante, uma vez que pesquisas mostraram que a maioria dos projetos que fracassam têm como seu principal motivo o mal planejamento dos custos e cronograma. Esse planejamento é fortemente centrado na experiência e conhecimento adquiridos em projetos anteriores. Quanto maior a experiência do gerente do projeto, melhor ele será capaz de realizar as estimativas para o projeto corrente. Porém, o conhecimento do planejamento de prazos e custos de um gerente de projeto não pode permanecer no nível do indivíduo. Para que a organização evolua aprendendo com seus próprios erros e acertos, é necessário que o conhecimento seja gerenciado de forma a tornar possível sua captura, recuperação e futura utilização. Prover uma organização de capacidade para utilizar uma abordagem de planejamento de prazos e custos prática e eficaz é um ponto diferencial para a mesma diante das exigências mercadológicas atuais. O trabalho descrito neste artigo veio, exatamente, propor uma abordagem para o planejamento de prazos e custos nas organizações. Essa abordagem está inserida em um tipo especial de ambientes de desenvolvimento de software, chamados de Ambientes de Desenvolvimento de Software Orientados à Organização (ADSOrg). O trabalho aqui descrito encontra-se, assim, no contexto dos ADSOrg, englobando outras ferramentas de gerência de projetos com enfoque em gerência do conhecimento.

Os benefícios da abordagem proposta poderão ser avaliados em um procedimento de validação da ferramenta. Porém, a validação de uma ferramenta como a CustPlan implica em sua utilização em vários projetos o que excede em muito o tempo esperado para uma tese de mestrado. Portanto, a validação, como mencionado na seção 2, está sendo realizada no contexto do Projeto TABA englobando seu conjunto de ferramentas.

As principais perspectivas para trabalhos futuros são o refinamento da busca por projetos similares e o desenvolvimento do mecanismo de calibração do COCOMO II. Uma outra melhoria seria a disponibilização dos dados armazenados no repositório da organização referentes a projetos similares de forma mais expressiva para o gerente do projeto, para auxiliá-lo nas estimativas de novos projetos. Um exemplo desses dados seria os valores médios de prazo, esforço e custos utilizados em projetos classificados como pequenos, médios e grandes.

Uma observação importante que deve ser registrada no que diz respeito à eficiência das estimativas geradas por uma determinada técnica ou abordagem, é que o processo de realização estimativas é um processo de previsão e não de precisão, sendo assim, uma margem de erro deve ser determinada e as estimativas que estiverem um desvio menor ou igual a essa margem de erro devem ser consideradas acuradas. 


\section{Referências Bibliográficas}

[1] ABECKER, A., BERNADI, A., HINKELMANN, K. et al., 1998, “Toward a Technology for Organizational Memories”, IEEE Intelligent Systems, v. 13, May/June, pp. 4048.

[2] BOEHM, B. W., ABTS, C., BROWN, A.W., CHULANI, S., CLARK, B.K., HOROWITZ, E., MADACHY, R., REIFER, D., STEECE, B., 2000, "Software Cost Estimation with COCOMO II", Prentice Hall.

[3] ISO/IEC DTR 16326 - Software Engineering - Guide for the Application of ISO /IEC 12207 to Project Management, 1999.

[4] GARMUS, D., HERRON, D., 2001, "Function Point Analysis: Measurement Practices for Successful Software Projects", Addison Wesley.

[5] JONES, C., 2000, "Software Assessments, Benchmarks, and Best Practices", AddisonWesley Information Technology Series, pp. 657 .

[6] MARKKULA, M., 1999, "Knowledge Management in Software Engineering Projects", In: Proceedings of the $11^{\text {th }}$ International Conference on Software Engineering \& Knowledge Engineering, Kaiserslautern, Germany, Jun, pp. 20-27.

[7] MENDONÇA, M. G., SEAMAN, C.B., BASILI, V., KIM, Y., 2001, "A Prototype Experience Management System for a Software Consulting Organization”, Software Engineering and Knowledge Engineering - SEKE, Buenos Aires, Argentina, June.

[8] MURCH, R., 2000, “Project Management: Best Pratices for IT Professionals”, Prentice Hall.

[9] NBR ISO 10006 - Gestão da Qualidade: Diretrizes para Qualidade no Gerenciamento de Projetos, 2000.

[10] NBR ISO/IEC 12207 - Tecnologia de Informação - Ciclos de Vida de Software, 1998.

[11] O’LEARY, D.E., STUDER, R., 2001, “Knowledge Management: An Interdisciplinary Approach”, IEEE Intelligent Systems, Jan/Feb, pp. 24-25.

[12] PMBOK - Project Management Body of Knowledge, 2000, PMI - Project Management Institute.

[13] RUS, I., LINDVALL, M., 2002, “Knowledge Management in Software Engineering”, IEEE Software, v. 19, Issue: 3 , May/Jun, pp. 26 -38.

[14] SCHNAIDER, L. (2003) "Planejamento de Alocação de Recursos Humanos em Ambientes de Desenvolvimento de Software Orientados à Organização", Tese de M. Sc., COPPE/UFRJ, Rio de Janeiro, RJ, Brasil.

[15] VIllelA, K., TRAVASSOS, G.H., ROCHA, A.R., 2000, “Ambientes de Desenvolvimento de Software Orientados à Organização”, Publicação Técnica COPPE/UFRJ - ES530/00 Rio de Janeiro, RJ, Abril.

[16] VILlELA, K., TRAVASSOS, G.H., ROCHA, A.R., 2001, “Ambientes de Desenvolvimento de Software Orientados à Organização”, IDEAS'2001 - Workshop Ibero-americano de Ingeniería de Requisitos y Ambientes de Software, Jan Jose, Costa Rica, Abril.

[17] WANGENHEIM, C. G. V., LICHTNOW, D., WANGENHEIM , A.. V., 2001, “ $A$ Hybrid Approach for Corporate Memory Management Systems in Software R\&D Organizations", $13^{\text {th }}$ International Conference on Software Engineering and Knowledge Engineering - SEKE 2001, pp. 326-330. 